\title{
A cohort study: Myocardial infarction with non- obstructive coronary arteries (MINOCA) in an Irish tertiary centre
}

\author{
Laura Piggott ${ }^{*}$, Simon Piggott ${ }^{1}$, Mohmed Allam $^{2}$ and Tom Kiernan ${ }^{2}$ \\ ${ }^{1}$ Saint James's Hospital, Dublin, Ireland \\ ${ }^{2}$ University Hospital Limerick, Ireland
}

\begin{abstract}
Myocardial infarction with non-obstructed coronary arteries (MINOCA) represents a diagnostic and therapeutic challenge. Approximately $10 \%$ of patients presenting with acute MI do not have obstructive coronary artery disease on coronary angiogram as confirmed in several large Acute Myocardial Infarction (AMI) registries. A diagnosis of MINOCA can be introduced in patients with consistent features of MI and demonstrated nonobstructive coronary artery disease. With increased utilisation of coronary angiograms and high sensitivity troponin, the identification of MINOCA is encountered more frequently. The long-term clinical outcome and prognosis of this subgroup is not well known or understood. The aim of this retrospective cohort study was to evaluate patient demographics, diagnosis and the 12-month follow-up of 26 inpatients who presented with MINOCA in an Irish tertiary centre. The following inclusion criteria was used in the definition of MINOCA; elevated troponin biomarkers, clinical features of ischaemia (symptoms, ischaemic changes on electrocardiogram, new loss of viable myocardium or wall motion abnormality) evidence of intracoronary thrombus or a non-obstructive pattern angiographically. Acute myocarditis was the most common cause of MINOCA with unobstructive angiography in this cohort of patients. $15.3 \%(\mathrm{n}=4)$ had a recurrent hospital admission with similar presentation within 12 months of the primary event. Establishing appropriate diagnosis and identifying patient risks is essential to ensure patients receive both preventative therapy and appropriate treatment.
\end{abstract}

\section{Introduction}

Acute myocardial infarction with unobstructed $(\leq 50 \%$ stenosis $)$ coronary arteries (MINOCA) has a prevalence of approximately $10 \%$ of patients with suspected myocardial infarction [1,2]. MINOCA remains a serious concern due to the uncertain risk of major adverse coronary events (MACE) during follow-up. Over the past century, the management of AMI has evolved with troponin values, changes on electrocardiogram, early use of coronary angiography and reperfusion therapies forming the foundation of contemporary AMI management guidelines. In this context, the European Society of Cardiology recommends following three criteria for the diagnosis of MINOCA [3].

\section{AMI criteria.}

a. Positive cardiac biomarker such as Troponin with at least one value above the 99th percentile upper reference limit.

b. Clinical evidence of ischaemia, including any of the following:

i. Ischaemic symptoms (chest pain/shortness of breath)

ii. Ischaemic electrocardiogram (ECG) changes (ST segment changes or new left bundle branch block)

iii. New pathological Q waves

iv. New regional wall motion abnormality

v. New loss of viable myocardium.

2. Absence of obstructive coronary artery disease or CAD ( $\geq 50 \%$ stenosis).

3. No clinically obvious cause for acute presentation.
These diagnostic criteria combine the current definitions of AMI and unobstructive CAD $[3,4]$. In this study, unobstructed coronary arteries are defined as stenosis $\leq 50 \%$ in any potential infarct related artery. This includes both mild CAD (stenosis $>30 \%$ but $\leq 50 \%$ ) and normal coronary arteries (no stenosis $>30 \%$ ) [5]. At present, MINOCA is a common clinical situation with an uncertain prognosis, however, it has been associated with lower rates of adverse clinical outcomes in various studies [6,7]. Establishing a definitive diagnosis in this subgroup remains challenging. Differential diagnoses for MINOCA would include MI related and non-MI related causes [8]. MI related causes include plaque rupture or erosion with spontaneous recanalization, coronary spasm, spontaneous coronary dissection, paradoxical coronary embolism, hypercoagulable states and demand ischaemia. In contrast, non-MI related causes of MINOCA include myocarditis, stress cardiomyopathy, dilated cardiomyopathy, hypertrophic cardiomyopathy, pulmonary embolism (PE), microvascular dysfunction (syndrome $\mathrm{X}$ ) and connective tissue disease $[9,10]$. For such patients, determining the correct diagnosis has important therapeutic and prognostic implications.

${ }^{\star}$ Correspondence to: Laura Piggott, Saint James's Hospital, Dublin, Ireland, E-mail: laurampiggott@gmail.com

Key words: acute myocardial infarction, myocarditis, tako-tsubo cardiomyopathy, angiography, cardiac magnetic resonance imaging, normal coronary arteries, nonobstructive coronary artery disease

Received: September 14, 2020; Accepted: September 24, 2020; Published: September 30, 2020 
Confirming a definitive diagnosis often involves a multi-modality approach with both invasive and non-invasive investigations playing a role. Although troponin is a sensitive and specific biomarker of myocardial necrosis it cannot determine the mechanism of damage. Cardiac troponins (cTn) I and T are organ specific, but not disease specific biomarkers [11]. Therefore, they may be elevated in both coronary and non-coronary aetiologies. However, use of imaging such as cardiac magnetic resonance (CMR) acts as a valuable tool to guide diagnosis and adapt management [12].

CMR is a comprehensive, non-invasive technique that has the ability to distinguish ischemic from non-ischaemic cardiac disease in the presence of normal angiogram [13]. It allows the assessment of cardiac anatomy, functional analysis and tissue characterization [14]. In this context, CMR results can lead to adaptation of management strategy and secondary prevention. As a technique, CMR plays an increasingly significant role in the diagnosis of various cardiovascular diseases [15]. Notably, literature has shown CMR may only identify a diagnosis in up to $95 \%$ of patients with ischaemic symptoms, an elevated troponin and unobstructed coronary arteries [16,17].

In this study, we sought to determine the prevalence of the different aetiological diagnoses in patients presenting with acute chest pain syndrome, elevated troponin concentration and unobstructed coronary arteries and their demographics. Additionally, data is sparse regarding baseline characteristics and the clinical outcomes of this patient group. In such case, the objective of this study was to examine the patient cohort establishing characteristics and both the diagnosis and adverse clinical outcomes of each patient.

\section{Methods}

This retrospective cohort study looked at a five-month period between September 2015 and January 2016 at University Hospital Limerick. All 146 patients with suspected AMI who underwent coronary angiogram were included in the study. Of these, 26 (17.8\%) had non-obstructive coronary artery disease (CAD) on assessment, defined in this study as $\leq 50 \%$ stenosis. From this cohort, all 26 patients met the following inclusion criteria leading to a diagnosis of MINOCA: (a) clinical evidence of ischaemia; ischaemic symptoms or electrocardiogram changes (new pathological Q waves, ST segment or $\mathrm{T}$ wave abnormalities), (b) raised troponin I levels, (c) unobstructed C on angiogram and (d) no apparent cause for presentation. 16 patients (61.5\%) received CMRI to guide diagnosis. Data collection included baseline characteristics, ECG on presentation, laboratory profile, GRACE score [18] and cardiac imaging results (Figure 1). Adverse

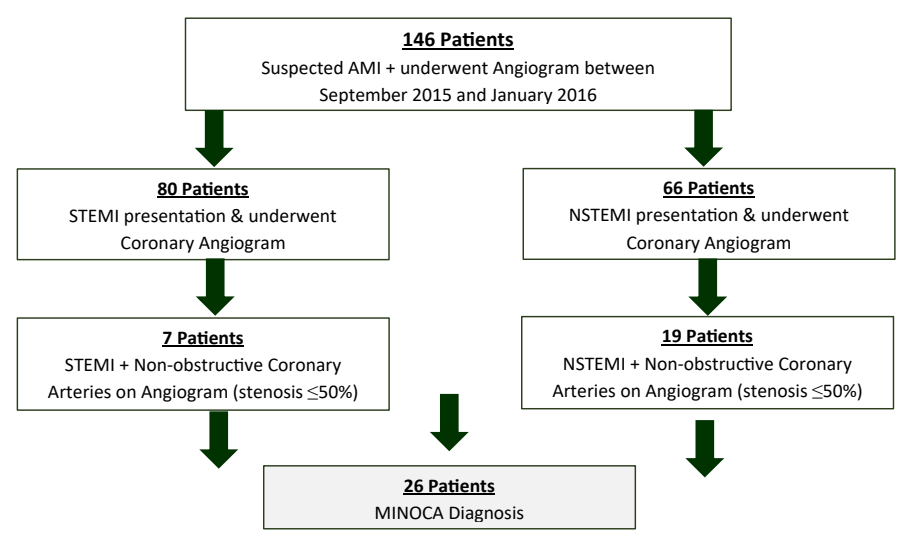

Figure 1. Selection process of patient cohort outcomes were recorded with 1-year follow up including hospital readmission, development of heart failure and overall mortality. All data was extracted from the National Citrix Portal and McKesson Clinical Management Software. Overall mortality was determined upon contacting a patient general practioner and accessing patient files. Statistical representation and analysis were completed using both Microsoft Excel and SPSS software. A p-value $\leq 0.05$ was considered statistically significant. Ethical approval for the study was obtained from the University Hospital Limericks Research Ethics Committee (113_15).

\section{Results}

Of a total of 146 patients admitted to the University Hospital Limerick with suspected ACS between September 2015 and January 2016, 80 presented as a ST elevation MI (STEMI) while 66 presented as a non-ST elevation MI (NSTEMI). Retrospectively, 26 (17.8\%) patients had an unobstructive pattern angiographically and met the predetermined inclusion criteria. Baseline characteristics recorded are summarized in (Table 1). The mean age was $51 \pm 20$ years. There was no gender predominance with $50 \%$ men $(n=13)$ and $50 \%$ women $(n=13)$. No patient had known pre-existing renal impairment. There was moderate prevalence of cardiovascular risk factors; hypertension (42.3\%), diabetes mellitus (15\%), hyperlipidaemia (7.6\%), smoking history (11.5\%) and a family history of ischaemic heart disease (15\%). 1 patient had known CAD with 1 stent in situ. No patient received thrombolytic therapy.

All 26 patients had an abnormal ECG on presentation as illustrated in table 2 . The most commonly detected abnormality was T-wave changes (46.1\%), followed by ST-segment depression (26.9\%) and ST-segment elevation (26.9\%). No patient had supraventricular or ventricular tachycardia on presentation. All patients received transthoracic echo that highlighted $69.3 \%$ of patients had an ejection fraction between $50-55 \% .61 .5 \%$ of patients $(n=16)$ received CMR to help ascertain diagnosis. 1 patient received a CT pulmonary angiography and was subsequently diagnosed with a pulmonary embolism. 1 patient received optical coherence tomography (OCT).

CMR was used in $61.5 \%(n=16)$ of the patient cohort. CMR revealed the aetiological diagnosis of $75 \%$ of these selected patients $(n=12)$. The details of the differential diagnosis are summarized in table 2. A diagnosis of acute myocarditis was the most frequently encountered in $31.3 \%(n=5)$ of patients, following the trend of previous studies $[12,19]$. Other diagnosis includes acute MI 25\% ( $\mathrm{n}=4)$, TTC $12.5 \%(\mathrm{n}=2)$ and HOCM $6.2 \%(n=1)$. In the 4 remaining patients, no new diagnosis was made - there was no impaired left ventricular function or detectable myocardial necrosis (Table 3 ).

Follow-up up information was available for 25 of 26 patients at 1 year. No alternate diagnosis was made for any patient during followup. 1-year follow-up adverse clinical events are summarized in table 4. No overall or cardiac mortality or congestive cardiac failure was recorded within this period. Four patients (15.3\%) were hospitalized during the follow up; Three patients (11.5\%) from the myocarditis group suffered from recurrence of chest pain and were readmitted at this time, there were no MACE observed. 1 patient from the TTC group also suffered from recurrence of chest pain and was readmitted, however, no MACE was recorded. Among those patients with normal CMRI results, hypertrophic cardiomyopathy and myocardial infarct no adverse clinical outcomes were observed during follow-up. 
Table 1. Baseline characteristics

\begin{tabular}{|l|l|}
\hline Characteristic & Value (n=26) \\
\hline Age (years) & $51( \pm 20 \mathrm{SD})$ \\
\hline Male & $13(50)$ \\
\hline Female & $13(50)$ \\
\hline Hypertension & $11(42.3)$ \\
\hline Diabetes mellitus & $4(15)$ \\
\hline Hyperlipidaemia & $2(7.6)$ \\
\hline History of smoking & $3(11.5)$ \\
\hline Previous IHD/PCI & $1(3.8)$ \\
\hline Family History IHD & $4(15)$ \\
\hline Renal Impairment & 0 \\
\hline
\end{tabular}

Table 2. Baseline investigations

\begin{tabular}{|l|l|}
\hline Characteristic & Value $(\mathbf{n}=\mathbf{2 6})$ \\
\hline ECG & \\
\hline ST segment elevation & $7(26.9)$ \\
\hline ST segment depression & $7(26.9)$ \\
\hline T wave changes & $12(46.2)$ \\
\hline cTn I on admission & $285.96 \mathrm{ng} / \mathrm{dl}$ \\
\hline Ejection Fraction & \\
\hline$>55 \%$ & $3(11.5)$ \\
\hline $50-55 \%$ & $18(69.3)$ \\
\hline $35-50 \%$ & $3(11.5)$ \\
\hline$<35 \%$ & $2(7.7)$ \\
\hline GRACE Score (Mean) & 100.4 \\
\hline
\end{tabular}

Table 3. Cardiac magnetic resonance imaging CMR diagnosis

\begin{tabular}{|l|l|l|l|}
\hline CMR Diagnosis & $\begin{array}{l}\text { Chopard et al. } \\
\mathbf{2 0 1 1}\end{array}$ & Chu et al. 2015 & Current Study \\
\hline & $(\mathbf{n}=\mathbf{8 7})^{[12]}$ & $\mathbf{( n = 3 2 ) ^ { [ 1 7 ] }}$ & $(\mathbf{n}=\mathbf{1 6})$ \\
\hline Tako-tsubo Cardiomyopathy & $10(11.5 \%)$ & $2(6.3 \%)$ & $2(12.5 \%)$ \\
\hline Hypertrophic Cardiomyopathy & 0 & $2(6.3 \%)$ & $1(6.2 \%)$ \\
\hline Acute Myocarditis & $23(26.4 \%)$ & $16(50 \%)$ & $5(31.3 \%)$ \\
\hline Myocardial Infarct & $22(22.7 \%)$ & $5(15.5 \%)$ & $4(25 \%)$ \\
\hline Normal CMR & $32(36.7 \%)$ & $2(6.3 \%)$ & $4(25 \%)$ \\
\hline
\end{tabular}

Table 4. Clinical outcomes

\begin{tabular}{|l|l|}
\hline Variable & N (\%) \\
\hline Overall Mortality & 0 \\
\hline Cardiac Mortality & 0 \\
\hline MACE & 0 \\
\hline Recurrence of symptoms & $4(15.3 \%)$ \\
\hline Hospital readmission & $4(15.3 \%)$ \\
\hline Congestive Cardiac Failure & 0 \\
\hline
\end{tabular}

\section{Discussion}

MINOCA is a novel clinical entity that is being increasingly recognized throughout the world. There are multiple reasons why this working diagnosis was created as explained by Pasupathy et al. [8] Establishing a diagnosis of MINOCA is important to avoid diagnostic complacency and to prompt further evaluation of underlying aetiologies and long-term prognosis of these patients.

\section{Who is at risk of MINOCA?}

Studies by both Berger et al. and Shaw et al. found that the clinical characteristics of MINOCA patients varied in comparison to obstructive CAD patients. MINOCA patients have been found to be younger with only a slight male predominance [20,21]. Further, a recent systematic review pooled data from 13 studies of MINOCA patients, finding the mean age of presentation as 55 years and $40 \%$ women [22]. Notably, hyperlipidaemia was a common finding. These collective results were similar to the baseline characteristics found in this cohort study which highlighted a mean age of 51 years with an equal predominance between male and female patients. However, in contrast with the aforementioned systemic review, only 2 patients in this study $(7.7 \%)$ presented with hyperlipidaemia. It is known that obstructive CAD are usually older patients with a higher incidence of middle-aged males than females. This trend of clinical characteristics suggests that underlying factors leading to MINOCA differ from those underlying an obstructive CAD patient.

MINOCA patients may first present with either STEMI or NSTEMI findings on electrocardiogram. Our study showed a higher incidence of NSTEMI patients $73 \%(n=19)$ than STEMI findings $27 \%(n=7)$. This compliments the findings of previous studies including DeWoods landmark study which highlighted up to $90 \%$ of STEMI patients had obstructive CAD, whereas only $26 \%$ of NSTEMI patients exhibited obstructive CAD $[17,23]$. With these findings, it could be presumed that higher rates of NSTEMI patients would be diagnosed with MINOCA. A more recent analysis of patients with NSTEMI within the CRUSADE registry highlighted that female sex and younger age were both independent predictors of MINOCA [24].

\section{Investigations to guide diagnosis}

A variety of diagnostic testing is required to narrow the differential diagnosis in the context of MINOCA. Although the information gained from a patient's history and blood tests is valuable, often it can be too circumstantial to be used clinically [25]. For example, atherosclerosis rates are increased in those with associated risk factors; age, positive family history, smoking, hyperlipidaemia, diabetes and hypertension. Further, a hereditary thrombophilia screen may be warranted in those with a positive family history of thromboembolism and hypercoagulability - as many a $14 \%$ of MINOCA patients have an abnormality detected upon screening [22]. Lastly, routine blood tests may provide a direction for diagnosis, with elevated D-dimer levels in patients with a PE or elevated acute inflammatory markers in patients with infection or acute myocarditis. Due to the non-specific nature of these investigations, non-invasive imaging techniques such as echocardiogram and CMR are of paramount importance in the MINOCA patient group [25]. Currently, echocardiogram may be more readily available than CMR and offers useful data on myocardial function, but it is not as specific as CMR. In this study, $100 \%$ of patients received a trans thoracic echocardiogram echoing its availability, in comparison, $75 \%$ of the patient cohort received a CMR to help establish a definitive diagnosis. CMR may be ordered to exclude subendocardial infarction in the presence of a normal angiogram and in clinical situation where the cause of MINOCA has not yet been found.

Although CMRI may not provide a diagnosis for all MINOCA patients it is a useful tool in differentiating between potential etiological causes. A comprehensive CMR allows categorization of potential diagnoses, both MI- and non-MI-related [26]. Results provided include fibrosis (delayed enhancement imaging), inflammation (T2 weighted imaging) and myocardial functioning (cine imaging) [27]. Previous literature has concluded that CMR provided a specific diagnosis in up to $75 \%$ of MINOCA patients who received the procedure [28]. These findings compliment the results of this study in which $61.5 \%$ of patients received a CMR and a diagnosis was established in $75 \%$ of those patients. Chu et al. showed an even higher rate of diagnosis by CMR in up to $95 \%$ of patients [17].

When CMR findings are normal and there is no cause for MINOCA found, the following clinical pictures should be considered, coronary 
plaque disruption, thromboembolism and vasospastic angina. In this cohort study, $25 \%$ of patients who underwent CMR exhibited no abnormal findings. This rate of normal findings was mirrored in a previous study by Assomull et al, even with the use of high field 3-tesla imaging [29]. These rates may be explained by inadequate resolution of the CMR, disease recovery or an alternate aetiology that is undetectable on CMRI. Additionally, in the context of normal perfusion and normal angiography a recanalised thrombus or paradoxical embolus should be considered [25]. Notably, prognosis in the setting of normal CMR has been reported as favourable [12].

CMR is the benchmark non-invasive method of establishing a diagnosis of acute myocarditis, which is of great importance for MINOCA patients. A recent meta-analysis showed the prevalence of myocarditis in MINOCA patients of 33\% [30]. In this study, acute myocarditis was the most common cause of MINOCA, a finding that has been reflected across multiple studies [16,30]. Routine investigations such as C-reactive protein can be ordered to provide clues for this diagnosis and to prompt further non-invasive cardiac imaging to confirm in light of it consistently being the leading cause of MINOCA.

Further, coronary vasospasm may be excluded by performing provocative testing using Acetylcholine or Ergonovine. Vulnerable plaques can be investigated using either optical coherence tomography (OCT) or intravascular ultrasound (IVUS) during the patient's time in the catheterization lab - due to inability of coronary angiography to visualize vessel walls. However, use of OCT and IVUS can be limited with high costs, restricted expertise and time availability as well as additional time for the patient under radiation exposure. Only 1 patient in this study received OCT, presumably due to the lack of significant atheromatous plaques on angiography [25].

\section{Prognosis}

The prognosis of MINOCA patients remains unclear however it is likely to be heterogeneous given the variety in diverse aetiological factors responsible. A 2015 systemic review by Pasupathy et al. compiled data from 8 studies and reported the following: in-hospital-all-causemortality of $0.9 \%$ (95\% confidence interval: $0.5 \%, 1.3 \%$ ) and $4.7 \%$ ( $95 \%$ confidence interval: $2.6 \%, 6.9 \%$ ) at 12 months [22]. This cohort study highlighted an overall mortality of $0 \%$ at 12 months, although limitations including a small patient cohort group exist. A recent metaanalysis highlighted a favourable lowered rate of death and MACE in MINOCA patients compared to acute coronary syndrome patients with obstructive $\mathrm{CAD}$. Interestingly, it also reported no difference in prognosis were noted between zero stenosis versus mild stenosis (1-49\%) [31].

There has been a great degree of variation in the recording of adverse outcomes for this group of patients, however, Bugiardini et al. suggest that the prognosis of MINOCA is not as benign as was previously reported [32]. In a study by Chopard et al. $5.7 \%$ of patients experienced MACE but there was no death in 1 year follow up [12] mirroring our study's findings of no overall mortality in the patient cohort during follow-up. However, the greatest risk exists in the initial phase as highlighted by Elesber et al, which listed complications such as ventricular arrhythmia or cardiogenic shock as life threatening with the MINOCA clinical setting [33].

It is disconcerting that according to the Korean MI registry prospectively found that MINOCA patients have an equivalent 12 month-all-cause mortality to those with an AMI with single- or doublevessel CAD [34]. Moreover, a study by Grodzinsky et al. concluded that $25 \%$ of MINOCA patients continued to experience chest pain 12 months after the main event [35]. These results combined with $15.3 \%$ recurrence of symptoms and re-admissions in our selected cohort should prompt larger scale analysis of these patients to give direction to the treatment of these patients and to avoid unsafe dismissal of insignificant CAD.

\section{The future of MINOCA}

At present, MINOCA is a working diagnosis for which specific evaluation and therapies differ as per the underlying cause. Therefore, focus should be placed on the accuracy of diagnosis to prevent adverse outcomes as suggested by recent literature [36].

Further research is warranted in evaluating the most appropriate treatment for MINOCA patients, especially for those in whom no underlying aetiological cause could be identified - which remains unsatisfyingly high. Clinicians remain uncertain if these patients should be treated empirically (with introduction of a statin and aspirin), if a calcium channel blocker should be started on the presumption of coronary artery spasm or should provocative testing of coronary spasm be routinely introduced in the catheritization lab? [36]. These are questions that need answering through the expansion of literature. At present, there are no randomized trials addressing effective therapies for MINOCA patients. Large scale multicentre collaborative studies may be of most benefit to advance further understanding. However, Lindahl et al. have very recently published a pivotal insight into long-term medical management of MINOCA. Using a large cohort of MINOCA patients $(n=9466)$, the key findings of the nonrandomized study were as follows; (a) a reduced hazard ratio of MACE with the use of statins or angiotensin-converting enzyme inhibitors/angiotensin receptor blockers and (b) no significant benefit of dual antiplatelet therapy. This study is the first of its kind with data on potential beneficial therapies to help the prognosis of MINOCA patients [37].

Recognising MINOCA as a diagnostic entity has improved our understanding and quality of care of this disorder. It is well established that MINOCA has many potential aetiologies that need to be elucidated by adhering to a strict diagnostic agreed algorithm as has been proposed by the European Society of Cardiology and is outlined in figure 2. Once commonly accepted and utilised, a large-scale register would be a useful tool to provide reliable estimates of true prevalence and prognosis of MINOCA patients [1].

\section{Study limitations}

One limitation of this study is the small sample size. Further study limitations include the lack of a control group and the identification

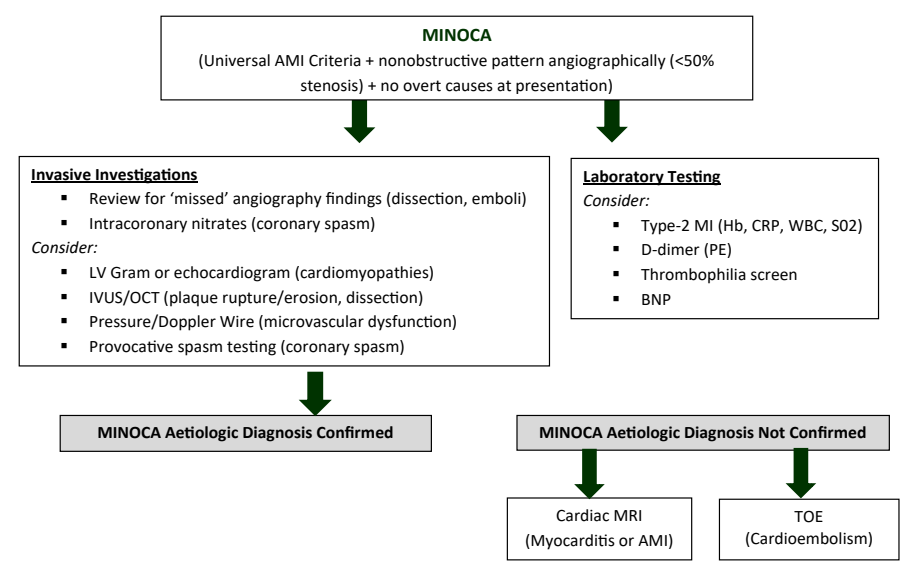

Figure 2. Recommended diagnostic and therapeutic algorithm for MINOCA patients 
of the cause of MINOCA was not identified in $25 \%$ of patients who received CMR.

\section{Conclusion}

Data from several large AMI registries suggest a prevalence between 5 and $25 \%$ highlighting the importance of MINOCA as a clinical entity [38]. Diagnosing patients with MINOCA remains challenging given the variation in possible aetiologies and so multimodalities are of continued importance. The novel diagnostic algorithm as suggested by the European Society of Cardiology should be followed and more commonly accepted. With this advancement, multi-centre large scale registers and consequent clinical trials of diagnostic and therapeutic strategies could impact on both treatment and prognosis of MINOCA patients as well as a better understanding of predictive patient demographics.

\section{References}

1. Agewall S, Beltrame JF, Reynolds HR, Niessner A, Rosano G, et al. (2017) ESC working group position paper on myocardial infarction with non-obstructive coronary arteries. Eur Heart J 38: 143-153. [Crossref]

2. Mozaffarian D, Benjamin EJ, Alan S, Arnett DK, Blaha MJ, et al. (2016) Heart Disease and Stroke Statistics-2016 Update: A Report from the American Heart Association. Circulation 133: e360-368. [Crossref]

3. Lewis BS, Agewall S (2016) Annual report of the European Society of Cardiology Working Group on Cardiovascular Pharmacotherapy. Eur Heart $J$ Cardiovasc Pharmacother 2: 3-4. [Crossref]

4. Thygesen K, Alpert JS, Jaffe AS, Simoons ML, Chaitman BR, et al. (2012) Third universal definition of myocardial infarction. Glob Heart 20: 2551-2567. [Crossref]

5. Scanlon PJ, Faxon DP, Audet AM, Carabello B, Dehmer GJ, et al. (1999) ACC/AHA guidelines for coronary angiography. A report of the American College of Cardiology/ American Heart Association Task Force on practice guidelines (Committee on Coronary Angiography). Developed in collaboration with the Society for Cardiac Angiography and Interventions. J Am Coll Cardiol 33: 1756-1824 [Crossref]

6. Dokainish H, Pillai M, Murphy SA, DiBattiste PM, Schweiger MJ, et al. (2005) Prognostic implications of elevated troponin in patients with suspected acute coronary syndrome but no critical epicardial coronary disease: a TACTICS-TIMI-18 sub study. J Am Coll Cardiol 45: 19-24. [Crossref]

7. Patel MR, Chen AY, Peterson ED, Newby LK, Pollack CV, et al. (2006) Prevalence, predictors, and outcomes of patients with non-ST-segment elevation myocardial infarction and insignificant coronary artery disease: results from the Can Rapid risk stratification of Unstable angina patients Suppress Adverse outcomes with Early implementation of the ACC/AHA Guidelines (CRUSADE) initiative. Am Heart $J 152$ 641-647. [Crossref]

8. Pasupathy S, Tavella R, Beltrame JF (2016) The What, When, Who, Why, How and Where of Myocardial Infarction with Non-Obstructive Coronary Arteries (MINOCA). Circ J 80: 11-16. [Crossref]

9. Larson DM, Menssen KM, Sharkey SW, Duval S, Schwartz RS, et al. (2007) "Falsepositive" cardiac catheterization laboratory activation among patients with suspected ST-segment elevation myocardial infarction. JAMA 298: 2754-2760. [Crossref]

10. Mahajan N, Mehta Y, Rose M, Shani J, Lichstein E, et al. (2006) Elevated troponin level is not synonymous with myocardial infarction. Int J Cardiol 111: 442-449. [Crossref]

11. Babuin L, Jaffe AS (2005) Troponin: the biomarker of choice for the detection of cardiac injury. CMAJ 2005. 173: 1191-1202. [Crossref]

12. Chopard R, Jehl J, Dutheil J, Genon VD, Seronde MF, et al. (2011) Evolution of acute coronary syndrome with normal coronary arteries and normal cardiac magnetic resonance imaging. Arch Cardiovasc Dis 104: 509-517. [Crossref]

13. Saba L, Fellini F, De Filippo F (2015) Diagnostic value of contrast-enhanced cardiac magnetic resonance in patients with acute coronary syndrome with normal coronary arteries. Jpn J Radiol 33: 410-417. [Crossref]

14. Zaldumbide LE, David EP, Larena JA, del Castillo SV, Cuevas JRR, et al. (2009) The value of cardiac magnetic resonance in patients with acute coronary syndrome and normal coronary arteries. Rev Esp Cardiol 62: 976-983. [Crossref]
15. Bruder O, Schneider S, Nothnagel D, Dill T, Hombach V, et al. (2009) EuroCMR (European Cardiovascular Magnetic Resonance) registry: results of the German pilot phase. J Am Coll Cardiol 54: 1457-1466. [Crossref]

16. Leurent G, Langella B, Fougerou C, Lentz PA, Larralde A, et al. (2011) Diagnostic contributions of cardiac magnetic resonance imaging in patients presenting with elevated troponin, acute chest pain syndrome and unobstructed coronary arteries. Arch Cardiovasc Dis 104: 161-70. [Crossref]

17. Chu G, Zhang G, Zhu M, Zhang Z, Wu Y, et al. (2015) Acute One-Stop Cardiovascular Magnetic Resonance Imaging for Differential Diagnosis in Patients with Acute Coronary Syndrome and Unobstructed Coronary Arteries. Med Princ Pract 24: 325331. [Crossref]

18. Granger CB, Goldberg RJ, Dabbous O, Pieper KS, Eagle KA, et al. (2003) Predictors of hospital mortality in the global registry of acute coronary events. Arch Intern Med 163: 2345-2353. [Crossref]

19. Niccoli G, Scalone G, Crea F (2015) Acute myocardial infarction with no obstructive coronary atherosclerosis: mechanisms and management. Eur Heart $J$ 36: 475-481. [Crossref]

20. Berger JS, Elliott L, Gallup D, Roe M, Granger CB, et al. (2009) Sex differences in mortality following acute coronary syndromes. JAMA 302: 874-882. [Crossref]

21. Shaw LJ, Shaw RE, Merz CNB, Brindis RG, Klein LW, et al. (2008) Impact of ethnicity and gender differences on angiographic coronary artery disease prevalence and inhospital mortality in the American College of Cardiology-National Cardiovascular Data Registry. Circulation 117: 1787-1801 [Crossref]

22. Pasupathy S, Air T, Dreyer RP, Tavella R, Beltrame JF (2015) Systematic review of patients presenting with suspected myocardial infarction and nonobstructive coronary arteries. Circulation 131: 861-870. [Crossref]

23. DeWood MA, Stifter WF, Simpson CS, Spores J, Eugster GS, et al. (1986) Coronary arteriographic findings soon after non-Q-wave myocardial infarction. $N$ Engl $J$ Med 315: 417-423. [Crossref]

24. Gehrie ER, Reynolds HR, Chen AY, Neelon BH, Roe MT, et al. (2009) Characterization and outcomes of women and men with non-ST-segment elevation myocardia infarction and nonobstructive coronary artery disease: results from the Can Rapid Risk Stratification of Unstable Angina Patients Suppress Adverse Outcomes with Early Implementation of the ACC/AHA Guidelines (CRUSADE) quality improvement initiative. Am Heart J 2009. 158: 688-694. [Crossref]

25. Bekkers, SC, Smulders MW (2015) High biomarkers, but normal angiogram: what next? Heart Metab 67: 15-20.

26. Mahrholdt H, Wagner A, Judd RM, Sechtem U, Kim RJ (2005) Delayed enhancement cardiovascular magnetic resonance assessment of non-ischaemic cardiomyopathies. Eur Heart J 26: 1461-1474. [Crossref]

27. Proctor RD, Shambrook JS, McParland P, Peebles CR, Brown IW, et al. (2011) Imaging hypertrophic heart diseases with cardiovascular MR. Clin Radiol 66: 176-186.

28. Gerbaud E, Harcaut E, Coste P, Erickson M, Lederlin M, et al. (2012) Cardiac magnetic resonance imaging for the diagnosis of patients presenting with chest pain, raised troponin, and unobstructed coronary arteries. Int J Cardiovasc Imaging 28: 783-794. [Crossref]

29. Assomull RG, Lyne JC, Keenan N, Gulati A, Bunce NH, et al. (2007) The role of cardiovascular magnetic resonance in patients presenting with chest pain, raised troponin, and unobstructed coronary arteries. Eur Heart J 28:1242-1249. [Crossref]

30. Tornvall P, Gerbaud E, Behaghel A, Chopard R, Collste O, et al. (2015) Myocarditis or "true" infarction by cardiac magnetic resonance in patients with a clinical diagnosis of myocardial infarction without obstructive coronary disease: A meta-analysis of individual patient data. Atherosclerosis 241: 87-91. [Crossref]

31. Pizzi C, Xhyheri B, Costa GM, Faustino M, Flacco ME, et al. (2016) Nonobstructive Versus Obstructive Coronary Artery Disease in Acute Coronary Syndrome: A MetaAnalysis. J Am Heart Assoc 5: e004185. [Crossref]

32. Bugiardini R, Merz CNB (2005) Angina with "normal" coronary arteries: a changing philosophy. JAMA 293: 477-484. [Crossref]

33. Elesber AA, Prasad A, Lennon RJ, Wright RS, Lerman A, et al. (2007) Four-year recurrence rate and prognosis of the apical ballooning syndrome. $\mathrm{J} \mathrm{Am} \mathrm{Coll} \mathrm{Cardiol}$ 50: 448-452. [Crossref]

34. Kang WY, Jeong MH, Ahn YK, Kim JH, Chae SC, et al. (2011) Are patients with angiographically near-normal coronary arteries who present as acute myocardial infarction actually safe? Int $J$ Cardiol 146: 207-212. [Crossref] 
Piggott L (2021) A cohort study: Myocardial infarction with non-obstructive coronary arteries (MINOCA) in an Irish tertiary centre

35. Grodzinsky A, Arnold SV, Gosch K, Spertus JA, Foody JM, et al. (2015) Angina Frequency After Acute Myocardial Infarction in Patients Without Obstructive Coronary Artery Disease. Eur Heart J Qual Care Clin Outcomes 1: 92-99. [Crossref]

36. Pasupathy S, Tavella R, Beltrame JF (2017) Myocardial Infarction with Nonobstructive Coronary Arteries (MINOCA): The Past, Present, and Future Management. Circulation 135: 1490-1493. [Crossref]
37. Lindahl B, Baron T, Erlinge D, Hadziosmanovic N, Nordenskjöld A, et al. (2017) Medical Therapy for Secondary Prevention and Long-Term Outcome in Patients with Myocardial Infarction with Nonobstructive Coronary Artery Disease. Circulation 135 . 1481-1489. [Crossref]

38. Bugiardini R, Manfrini O, De Ferrari GM (2006) Unanswered questions for management of acute coronary syndrome: risk stratification of patients with minimal disease or normal findings on coronary angiography. Arch Intern Med 166: 1391-1395. [Crossref]

Copyright: $\odot 2021$ Piggott L. This is an open-access article distributed under the terms of the Creative Commons Attribution License, which permits unrestricted use, distribution, and reproduction in any medium, provided the original author and source are credited. 